\title{
A Review of the "Open" and "Closed" Circulatory Systems: New Terminology for Complex Invertebrate Circulatory Systems in Light of Current Findings
}

\author{
Carl L. Reiber and Iain J. McGaw \\ Integrative Physiology Section, School of Life Sciences, University of Nevada at Las Vegas, Las Vegas, NV 89154, USA \\ Correspondence should be addressed to Carl L. Reiber, carl.reiber@unlv.edu
}

Received 3 July 2008; Accepted 29 October 2008

Recommended by Stephen Tobe

\begin{abstract}
Invertebrate cardiovascular systems have historically been viewed as sluggish, poorly regulated, and "open", where blood bathes the tissues directly as it moves through a system of ill-defined sinuses and/or lacunae without an endothelial boundary. When examining cardiovascular/circulatory morphology and physiology in a broader evolutionary context, one can question the very nature of the definition of a "closed" versus "open" circulatory system. Viewed in this context a number of invertebrates have evolved incomplete or even completely cell-lined vessels and or lacunae with a highly branched vasculature that allows for the production of significant driving pressures and flows to meet relatively high metabolic demands driven by active life styles. In light of our current understanding of invertebrate cardiovascular systems and their paralleled complexity to vertebrate systems, a number of long established paradigms must be questioned and new definitions presented to better align our understanding of the nature of "open" versus "closed" cardiovascular systems.
\end{abstract}

Copyright (C) 2009 C. L. Reiber and I. J. McGaw. This is an open access article distributed under the Creative Commons Attribution License, which permits unrestricted use, distribution, and reproduction in any medium, provided the original work is properly cited.

\section{Introduction}

Historically, the invertebrate cardiovascular system has been viewed as one that is "open," sluggish, and poorly regulated, where blood bathes the tissues directly as it moves through a system of sinuses and/or lacunae without an endothelial boundary. In light of current findings, our view of the invertebrate circulatory system and with particular emphasis on the more complex invertebrate systems must change. The intent of this article is to draw parallels between the more complex invertebrate circulatory architectures and vertebrate systems using a limited and selective review of the literature to emphasize structural and physiological complexities. We provide support for arguments leading to a rethinking of the complexity of invertebrate circulatory systems as well as new definitions for terms commonly used to describe various circulatory architectures.

When cardiovascular/circulatory morphology and physiology are examined in a broad evolutionary context, the very nature of a "closed" versus "open" circulatory system can be questioned. Viewed in this context a number of invertebrates have evolved incomplete or even completely cell-lined vessels and or lacunae with a highly branched vasculature that allows for the production of significant driving pressures and flows to meet relatively high metabolic demands driven by active life styles. These invertebrate cardiovascular systems have well-developed muscular pumps with complex regulatory mechanisms that facilitate a dynamic range of responses to changing metabolic and environmental demands, and thus have allowed the exploitation of both aquatic and terrestrial ecosystems. In fact, many parallels can be drawn between the well-developed cardiovascular systems of lower vertebrates to those of decapod crustacea and cephalopod Molluscs. In light of our greater understanding of invertebrate cardiovascular systems and their paralleled complexity to vertebrate systems, a number of long established paradigms must be questioned and new definitions presented to better align our understanding of the nature of "open" versus "closed" cardiovascular systems. 


\section{Why the Classic Definition of "Open" and "Closed" Circulatory Systems Must be Rethought?}

The classic view of an open circulatory system is based on the image of pseudocoelomic or coelomic fluid bathing the tissues directly; this fluid is circulated throughout the coelom via the actions of the body wall musculature and animal movements. A second and somewhat more robust image of an open system is that of a dorsally located muscular vessel or heart sitting within a hemocoel, pumping hemolymph through anterior and/or posterior aortic vessels. These vessels end abruptly where their contents move into the coelom or other large space where gas, nutrient, and waste exchange take place directly between the cells (tissues) and hemolymph (or lymph-at this point the fluid could be described as extracellular fluid). Hemolymph then moves through venous sinuses or simply through the coelom and into a pericardial sinus, through cardiac ostia and into the heart for recirculation. Indeed both of these views are technically correct, yet convey the idea of a primitive, poorly designed and regulated cardiovascular system that is unable to sustain higher metabolic demands (Figure 1(a)).

Looking at the issue from the other side, our standard view of a closed circulatory system is based on a system where a multichambered muscular heart pumps blood through parallel systemic and pulmonary circuits simultaneously (Figure 1(c)). Blood is pumped into major elastic arteries (the aorta and large arteries), which then flows into medium and small smooth muscle-based vessels and then into arterioles, which supply the capillary circulation. At the capillary level, gas, nutrient, and waste exchange take place between blood and tissues across an endothelial layer. Venous blood then returns to the heart via, venules, small and medium veins, and finally back into the heart via the vena cava. In the closed circulatory system at no point does the blood leave the confines of the vascular endothelia and as such there is a clear distinction between blood and lymph [1-3].

While the descriptions above do represent accurate depictions of the circulatory systems of worm-like invertebrates and mammals, respectively, they do not provide the necessary depth and breadth of information required to understand the subtle yet significant "shades of grey" of the continuum from the invertebrate "open" and vertebrate "closed" circulatory architecture (Figure 1(b)). An exhaustive phylogenetic review of cardiovascular morphologies is not necessary to make this point clear. A few well-described examples from specific taxa can be used to illustrate the complexity of the issue and dramatically point out the shortcomings of the existing definitions.

\section{The Typical Invertebrate "Open" Circulatory System: The Annelid Blood-Vascular System}

Members of the phyla Annelida contain some of the most complex examples of worm-like invertebrates [5-7]. The segmented annelids have evolved several mechanisms in order to enhance convective transport between internal compartments. The most primitive of these being the development of a coelom and coelomic circulation followed by the development of intracellular iron-based oxygen binding pigments (hemoglobins), and the most advanced being a fairly well-developed blood-vascular system $[8,9]$. In the smaller annelids there are few cardio-respiratory adaptations, however; in the larger and/or more active worms, such as the polycheates, a complex vasculature has evolved and in the more active giant Australian earthworm (Oligocheata) a defined heart augments the movement of blood through a well-developed vasculature (Figure 2) [10].

While there are many anatomical variations observed in the cardiovascular system of annelids that appear to have evolved due to activity patterns, feeding behaviors and environment, some of the most complex systems are seen in the class Polychaeta. The general pattern of circulation in polycheate worms starts with a dorsal vessel that runs just above the digestive tract (Figure 3 ). Blood flows anteriorly where the dorsal vessel anastomes with a ventral vessel either directly or by several parallel connecting vessels. The ventral vessel runs under the digestive tract and carries blood posteriorly. Each segment of the animal receives a pair of parapodial blood vessels that arise from the ventral vessel. The segmental parapodial vessels supply the parapodia, the body wall (integument), and the nephridia and give rise to intestinal vessels that supply the gut. Blood moves from the ventral vessel through the parapodial system and returns to the dorsal vessel through a corresponding segmental pair of dorsal parapodial vessels (Figure 3 ). When gills are present and integrated with the blood vascular system (as opposed to being perfused with coelomic fluid) they contain both afferent and efferent vessels (Ruppert and Barnes 1991). Pressures are generated by peristaltic waves of contractions through the dorsal vessels. These blood vessels and their associated blood sinuses do not contain an endothelium but are lined by only the basal lamina of overlying cells (Figure 3) (Brusca and Brusca 1990).

\section{The Atypical Invertebrate "Closed" Circulatory System: The Cardiovascular System of the Cephalopod Mollusca}

The molluscan cardiovascular system has evolved extensive vascular networks with efficient centralized pumps (e.g., true hearts) that function in an integrated fashion with a variety of other physiological systems. The functional significance of these complex vessels is seen in the highly active Cephalopod Molluscs, which show the most extensive evolution and specialization of the cardiovascular system (Figure 4). Blood is driven at high pressures by the heart through a cell-lined (closed circulatory system) complex circulatory system that is able to sustain metabolic rates almost equivalent to some vertebrates $[13,14]$. To sustain such high oxygen uptake rates, paired branchial hearts have evolved to pump venous blood through the gills, after which the arterial blood flows to the ventricle where it is pumped to the systemic circuit. Functionally, the cephalopods have evolved a multichambered 


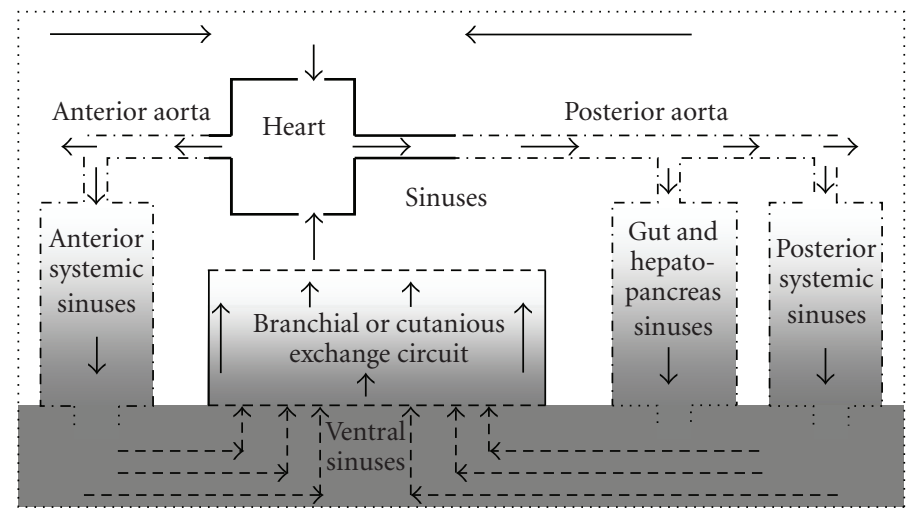

(a)

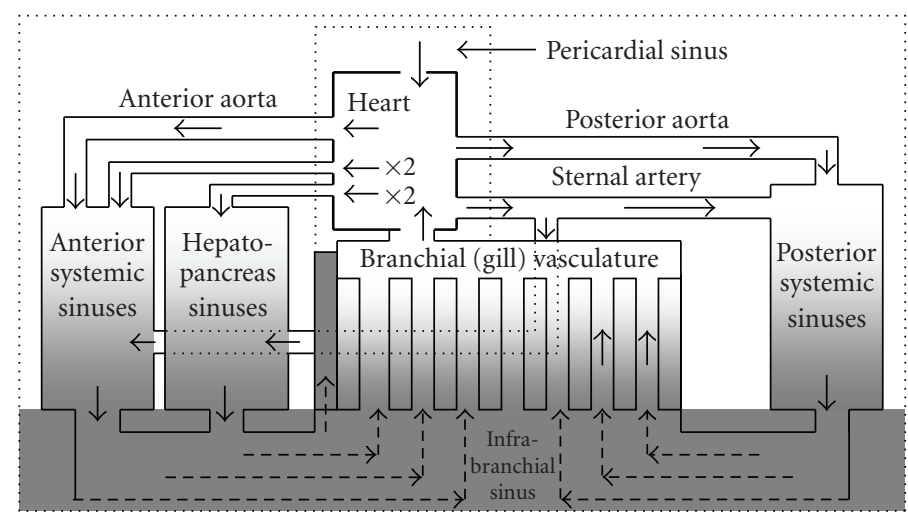

(b)

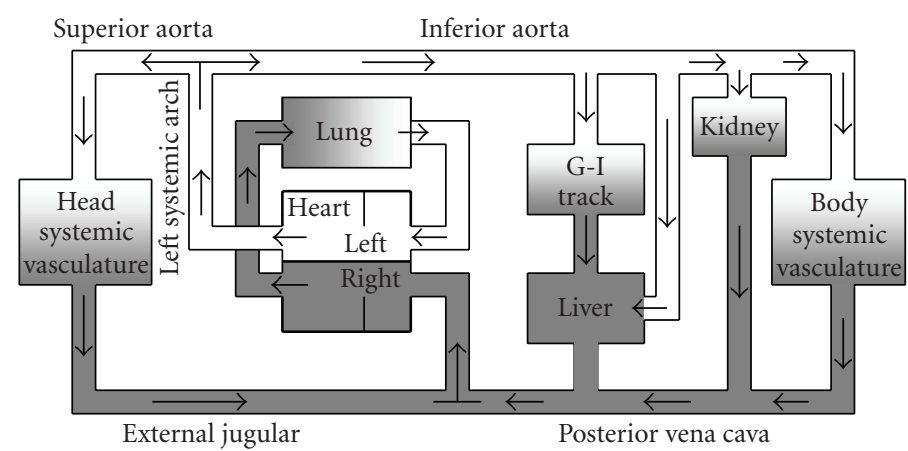

(c)

FIGURE 1: Schematics of: (a) a classically defined "open" circulatory system (as seen in may lower invertebrates), (b) a circulatory system that is highly complex with capillary like vessels, a partially lined vasculature yet contains vascular sinuses which classically has been defined as "open" yet should be categorized as an "incompletely closed" circulatory system. (c) A classically defined "closed" circulatory system (as seen in mammals and other higher vertebrates) ((c) adapted from Whithers [1]). (Solid lines represent defined vessels or a muscular pump or heart. Dashed lines represent sinus based systems and/or vessels lacking a defined lining. Arrows represent general patterns of blood flow. Background color is a general indicator of arterial versus venous hemolymph or blood).

heart capable of maintaining separation between venous and arterial blood and regulating branchial and systemic circulations. Additionally, this group of animals has developed the cardio-respiratory regulatory mechanisms needed to integrate cardiovascular and ventilatory performance with metabolic demands [15]. The anatomical complexity of the cardiovascular system, along with the development of capillary-like exchange vessels, an endothelia-like vascular lining and the appropriate regulatory mechanisms appears to have been selected for in this group by increased activity patterns associated with predatory behavior, swimming, and jet propulsion $[12,13]$.

While the cardiovascular systems of these more active cephalopods are quite robust and seem to exhibit convergent evolution with some vertebrates, in terms of their vascular complexity, there is a great deal of discrepancy in reports as to 


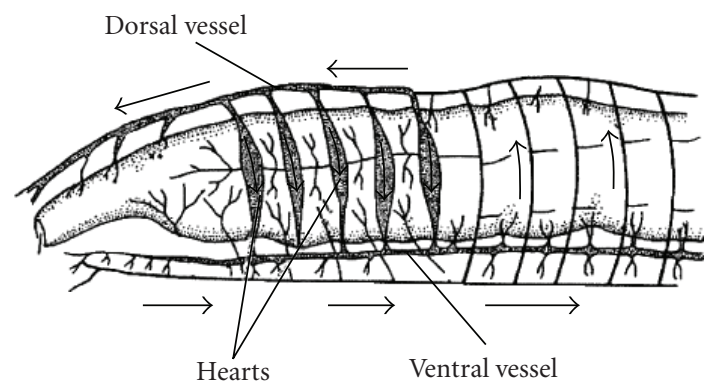

(a)

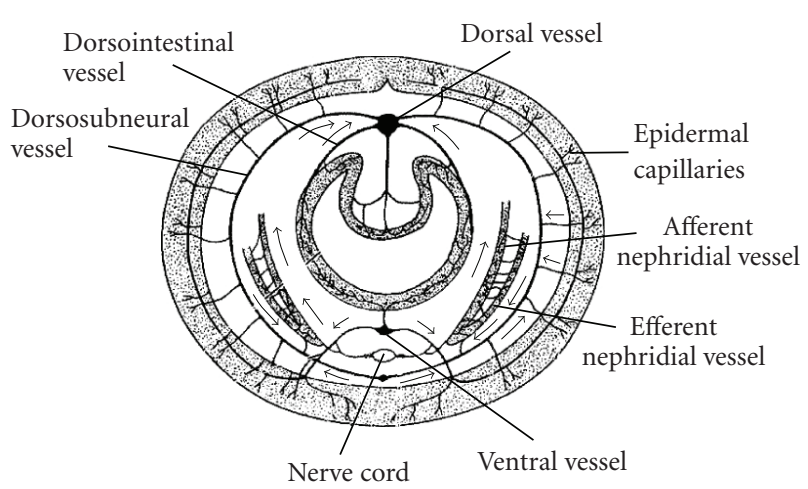

(b)

Figure 2: The annelid circulatory system, though a low pressure system, contains contractile vessels for pumps and a highly branched vascular system. It lacks an endothelial lining. (a) Longitudinal section, (b) cross-section of the earthworm (Lumbricus) (adapted from R. C. Brusca and G. J. Brusca, [4]).

the nature of the endothelia-like lining of the vessels and the degree to which tissues are perfused. It would appear that the cephalopod vascular lining is quite permeable and may not serve as a selective barrier as in the vertebrates, which could by some definitions make the cephalopod vascular system more "open" than "closed" [14, 17-19]. Yet based on a more functional or physiological definition, cephalopods exhibit a "closed" vascular system.

This leads to the question: why have cephalopods evolved both complete and/or incomplete endothelial-like lined vascular systems? It can be hypothesized that the invertebrate vascular lining has evolved for reasons more to do with hemodynamics and maintaining laminar flow, than the array of functions ascribed to the vertebrate vascular endothelia. The evolutionary origin of the invertebrate vascular system and its lining are derived from the coelom, yet few invertebrate taxa exhibit a vascular endothelium [20]. Those that do are more active with higher metabolic demands and also have well-developed centralized pumps, an extensively branched vasculature with "capillary-like" vessels and relatively high blood pressures.

Laminar flow is required to minimize the energy needed to move blood through these complex vascular systems. Laminar flow through a cylindrical tube can be predicted based on vessel diameter, mean blood velocity, and blood density and viscosity (Reynold's number). However, if there are sudden variations in vessel diameter or irregularities in

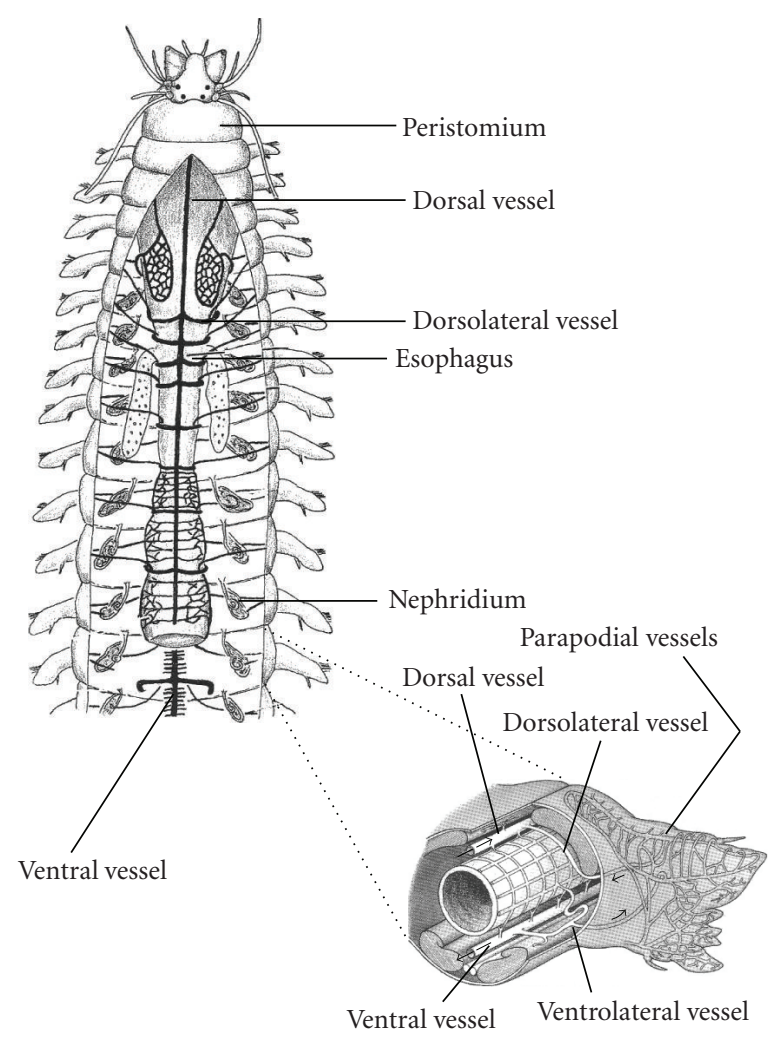

FIGURE 3: The fine structure of the annelid (Polycheata) circulatory system. The center diagram shows the general anatomy of the segmentally based vascular system. A cross-section through the body wall of the polycheates is shown on the left with body wall, epidermal, and coelomic vessels identified. The highly branched parapodial vasculature is outlined on the right (adapted from $[11,12]$.

the vessels walls turbulent flow can result. In turbulent flow a significantly greater pressure is required to move a fluid through the vessels as compared to laminar flow. This is best exemplified by the fact that in turbulent flow the pressure drop is approximately proportional to the square of the flow rate as opposed to laminar flow where the pressure drop is proportional to the first power of the flow rate $[21,22]$.

It would require a robust heart and would be energetically inefficient to move blood in a turbulent pattern through a vasculature that changes shape abruptly and where the interiors of the vessels are not smooth as is seen in many lacunar systems. Thus to minimize the energy required to move blood through the cephalopod circulatory system it would be advantageous to evolve mechanisms to facilitate laminar flow and one of which could be the development of an endothelia-like lining [23].

\section{New Definitions and New Views of Old Circulatory Systems}

Our current view of circulatory system architectures fits into one of two catergories; an "open" system or a "closed" system. While there is relatively little variation within closed 


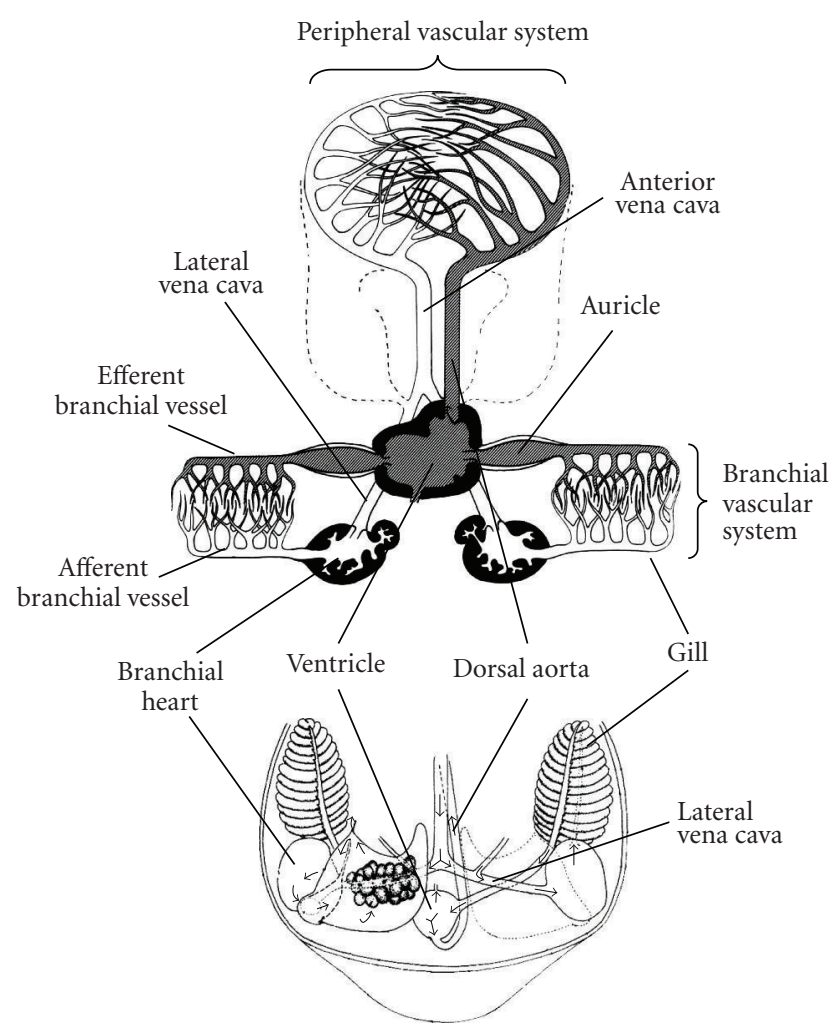

Figure 4: A generalized schematic of the cephalopod molluscs circulatory systems (top) and a more anatomically correct view (bottom), showing the well-developed hearts (a ventricle and two branchial hearts) and complex, endothelial-like lined vascular systems (peripheral and branchial). The cephalopod vascular system is considered "closed" with highly efficient hearts pumping blood at pressures similar to those seen in lower vertebrates (adapted from Smith and Boyle [16], Ruppert et al. [12]).

systems, the same cannot be said of open systems, which appear to vary greatly in complexity from a simple globular pump with no specialized vessels to a more-or-less complete circulatory system. Despite the presence of these "complete" open systems that appear to rival the complexity (both morphologically and physiologically) (Figure 1(b)) of the closed systems of lower vertebrates, several factors continue to define them as simply "open".

A major factor that defines a closed circulatory system as distinct from a "complete" open system is the presence of a proper endothelium lining the vessel walls. The circulatory systems of all vertebrates are completely lined by an endothelial cell layer as opposed to the invertebrates, cephalochordates, and tunicates. The vertebrate endothelium is defined as being a continuous sheet of mesodermally derived cells lining the vasculature (and in a broader context even more widely distributed throughout the body) are highly active with multiple functions and are heterogeneous in structure and function [24]. Functionally and most simply, the endothelium serves as a selective barrier separating the blood from the tissues (i.e., defining the three compartment system-blood, extracellular fluid [lymph], and intracellular fluid). A more modern and comprehensive view of endothelial function would include significant roles in hemodynamics, hemostasis, vasomotor tone, growth, and proliferation of other cells, antigen presentation, and metabolism of tissue or blood derived hormones [25]. For the purposes of this discussion, a functional barrier separating circulating blood from the tissues defines a closed circulatory system and as such can be considered the primary function of the endothelia or the cell-lined vasculature.

Unlike closed systems where blood and lymph are functionally separated by the endothelium, in truly open systems these two fluids are considered to mix freely and are thus termed hemolymph (blood and lymph). However, the presence or absence of hemolymph does not explicitly define a system as open or closed as comparative physiologists also define hemolymph based on the absence of defined cell lineages (red cells, thrombocytes, and leukocytes). Thus, one may have a cell-lined circulatory system that meets the definition of being closed yet contains hemolymph as seen to varying degrees in the cephalopods and crustaceans [4].

The definition of open versus closed is therefore based upon histological (endothelium) and cellular (hemolymph) terms rather than in physiological terms (functional). How can these two disparate views be reconciled given our new insights into the complexity of some "open" systems? If one also considers the circulatory system in physiological terms (which is its main function) then this allows a rethinking of the "open" versus "closed" definition with the addition of a new category-defined as an "incomplete closed system" as seen in the decapod crustaceans.

\section{An Example of the "Incomplete" Closed Circulatory System: The Decapod Crustacean}

Although there are more species of insects than any other group in the world and more individual nematodes, crustaceans exhibit a greater variation in form and diversity than any other animal phylum [28]. The decapod crustaceans have colonized a wide range of environments from the deep sea through the intertidal zone, and onto land. During the evolution of the invertebrates a number of key adaptations were responsible for their radiation. In crustaceans, the evolution of a segmental arterial system was a singular event that made the unique adaptive radiation of this group possible and the evolutionary innovation that allowed members of this group to become large and highly mobile [29].

Historically, the crustacean circulatory system has been considered open. However, during the past two decades our knowledge of the decapod crustacean circulatory system has increased substantially [26, 27, 29-32]. The muscular ventricle is housed inside a primer chamber, the pericardial sinus. Heart rate and stroke volume can be controlled independently via nervous input from the cardiac ganglion and CNS or by direct actions of neurohormones on the cardiac muscle $[29,32]$. This allows rapid modulation of cardiac output resulting in blood pressures that rival those of some fish and amphibians [33, 34]. Extrinsic 


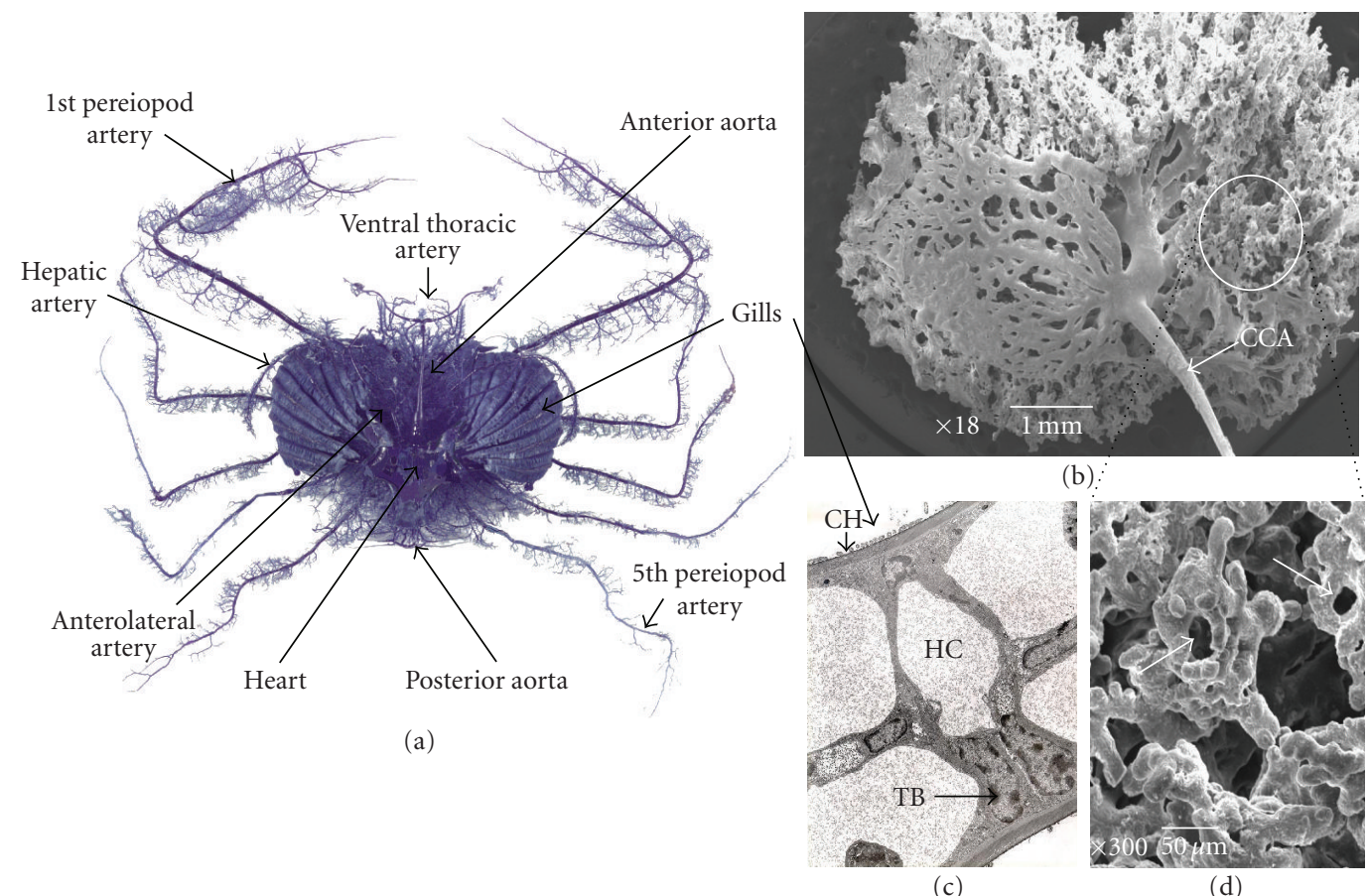

FIGURE 5: The cardiovascular system of decapod crustaceans is highly developed with a globular heart capable of delivering hemolymph at relatively high pressures and flows into capillary-like vessels supplying metabolically active tissues. This distribution system is dramatically illustrated (a) in a corrosion cast of the blue crab's circulatory system. The complexity of the decapod crustacean vasculature is seen in a corrosion cast of the antennal gland ((b) from McGaw and Reiber [26]) and a highly magnified image of the capillary like vessels serving this structure (d) (CCA-coelomosac artery) [27]. A transmission electron micrograph of a cross-section through the gills also clearly shows well-defined hemolymph channels that maximize branchial exchange ((c) from McGaw and Reiber [26]) (CH-chitinous exchange surface of lamellae; HC—hemolymph channels; PC—pillar cells).

control of cardiac function in vertebrate systems is primarily autonomic (sympathetic excitation and parasympathetic inhibition) layered upon intrinsic regulatory mechanisms. At the extrinsic level of control, parallel regulatory systems are seen in the neurogenic hearts of decapod crustaceans. Cardioacceleratory and cardioinhibitory nerves provide input to the cardiac ganglion, modulating the rate and force of myocardial contractions. Additionally, the pericardial organ, an endocrine organ located on the inner wall of the myocardium, releases a variety of neurohormones that can modulate heart rate and cardiac contractility [32].

Regional blood flow is regulated in closed vertebrate systems by the contraction or relaxation of vascular smooth muscle. Decapod crustaceans do not possess smooth muscle in the artery walls [35]; instead contraction or relaxation of a pair of muscular cardioarterial valves at the base of each arterial system [36] controls hemolymph flow through the arteries [37, 38]. A variety neurohormones have been shown to control regional hemolymph flow (see McGaw and McMahon [39], Wilkens [29], McGaw and Reiber [26]) either by direct actions on the cardioarterial valves or by altering downstream resistance of vessels [40, 41]. Such ability to modulate cardiac function and regional blood flow rivals that of vertebrate systems $[42,43]$.

In-line with physiological control mechanisms, the anatomy of the system is equally complex. Five arterial systems (seven individual vessels) originate from the heart, each splitting into smaller arteries and finally into capillarylike vessels that ramify within the tissues. Some of these vessels are similar in size (diameter-wise) to those of vertebrate capillaries and form a true closed loop within the brain [44, 45] and antennal gland [27] (Figure 5). Nevertheless, decapod crustaceans lack a complete venous system; instead the hemolymph collects in sinuses before flowing into large veins and back to the heart. In part, it is the presence of these sinuses that has defined the system as open. The sinuses were once considered to be "ill-defined spaces that almost defied successful demonstration" [46]. However, recent evidence has shown them to be more complex than previously described, forming a network of lacunae with a morphology similar to capillaries [27, 47], the only difference being the lack of a true endothelial lining. One hundred and fifty years ago Haeckel [48] proposed that no unbounded lacunae exist in the crustacean system. Major sinuses are bordered by fibrous connective tissue and the lacunae by basal lamina directly on the organ which they bathe [49]. The distinction between lacunae and capillary then becomes less distinct, suggesting a more organized structure. Thus, the definition of the open system of decapod crustaceans is really a histological term rather than a functional one. This then lends itself to an additional definition that would classify some of the highly complex open systems (in both decapod 
crustaceans but also others such as the cephalopods) as ones that are "incompletely closed" rather than open.

The decapod crustacean system exemplifies this and given the wide array of open systems the term "incompletely closed" would describe a complex system, with a muscular heart generating relatively high pressures, which delivers fluid through a complex series of vessels. This will clarify some of the confusion associated with the highly complex open systems with a complete series of vessels, versus those that are simple and sluggish with few associated vessels or control mechanisms.

\section{References}

[1] P. C. Whithers, Comparative Animal Physiology, Saunders College, Fort Worth, Tex, USA, 1992.

[2] W. W. Burggren and B. B. Keller, Development of Cardiovascular Systems, Cambridge University Press, Cambridge, UK, 1997.

[3] G. H. Satchell, Physiology and Form of Fish Circulation, Cambridge University Press, Cambridge, UK, 1991.

[4] R. C. Brusca and G. J. Brusca, Invertebrates, Sinauer Press, Sunderland, Mass, USA, 2nd edition, 2002.

[5] A. Toulmond, "Adaptations to extreme environmental hypoxia in water breathers," in Comparative Physiology of Environmental Adaptations, P. Dejours, Ed., vol. 2 of 8th ESCP Conference, Strasbourg, pp. 123-136, Karger, Basel, Switzerland, 1987.

[6] C. P. Mangum, J. M. Colacino, and T. L. Vandergon, "Oxygen binding of single red blood cells of the annelid bloodworm Glycera dibranchiata," Journal of Experimental Zoology, vol. 249, no. 2, pp. 144-149, 1989.

[7] D. Fritzsche and J. A. von Oertzen, "Metabolic responses to changing environmental conditions in the brackish water polychaetes Marenzelleria viridis and Hediste diversicolor," Marine Biology, vol. 121, no. 4, pp. 693-700, 1995.

[8] C. R. Vinson and J. Bonaventura, "Structure and oxygen equilibrium of the three coelomic cell hemoglobins of the Echiuran worm Thalassema mellita (conn)," Comparative Biochemistry and Physiology Part B, vol. 87, no. 2, pp. 361-366, 1987.

[9] P. DeJours and A. Toulmond, "Ventilatory reactions of the lugworm Arenicola marina (L.) to ambient water oxygenation changes: a possible mechanism," Physiological Zoology, vol. 61, no. 5, pp. 407-414, 1988.

[10] H.-O. Pörtner, "Anaerobic metabolism and changes in acidbase status: quantitative interrelationships and ${ }_{\mathrm{p}} \mathrm{H}$ regulation in the marine worm Sipunculus nudus," The Journal of Experimental Biology, vol. 131, no. 1, pp. 89-105, 1987.

[11] T. Nakao, "An electron microscopic study of the circulatory system in Nereis japonica," Journal of Morphology, vol. 144, no. 2, pp. 217-235, 1974.

[12] E. E. Ruppert, R. S. Fox, and R. D. Barns, Invertebrate Zoology: A Functional Evolutionary Approach, Brooks Cole, Belemont, Calif, USA, 7th edition, 2003.

[13] A. Packard, "Cephalopods and fish: the limits of convergence," Biological Reviews, vol. 47, no. 2, pp. 241-307, 1972.

[14] G. B. Bourne, J. R. Redmond, and D. D. Jorgensen, "Dynamics of the molluscan circulatory system: open versus closed," Physiological Zoology, vol. 63, no. 1, pp. 140-166, 1990.

[15] P. J. S. Smith, "Integrated cardiovascular control in the Mollusca," Physiological Zoology, vol. 63, no. 1, pp. 12-34, 1990.
[16] P. J. S. Smith and P. R. Boyle, "The cardiac innervation of Eledone cirrhosa (Lamarck) (Mollusca: Cephalopoda)," Philosophical Transactions of the Royal Society of London. Series B, vol. 300, no. 1101, pp. 493-511, 1983.

[17] V. C. Barber and P. Graziadei, "The fine structure of cephalopod blood vessels II. The vessels of the nervous system," Zeitschrift für Zellforschung und Mikroskopische Anatomie, vol. 77, no. 2, pp. 147-161, 1967.

[18] V. C. Barber and P. Graziadei, "The fine structure of cephalopod blood vessels III. Vessel innervation," Zeitschrift für Zellforschung und Mikroskopische Anatomie, vol. 77, no. 2, pp. 162-174, 1967.

[19] J. Browning, "Octopus microvasculature: permeability to ferritin and carbon," Tissue \& Cell, vol. 11, no. 2, pp. 371-383, 1979.

[20] J. Vagvolgyi, "On the origin of mollusks, the coelom and coelomic segmentation," Systematic Zoology, vol. 16, pp. 153$168,1967$.

[21] W. W. Nichols and M. F. O’Rourke, McDonald's Blood Flow in Arteries: Theoretic, Experimental and Clinical Principles, Lea and Febiger, Philadelphia, Pa, USA, 3rd edition, 1990.

[22] R. M. Berne and M. N. Levy, "The arterial system," in Cardiovascular Physiology, R. M. Berne and M. N. Levy, Eds., pp. 94-108, C.V. Mosby, Saint Louis, Mo, USA, 3rd edition, 1991.

[23] W. W. Burggren and C. L. Reiber, "Evolution of cardiovascular systems," in Endothelial Biomedicine, W. C. Aird, Ed., pp. 2949, Cambridge University Press, Cambridge, UK, 2007.

[24] W. C. Aird and M. D. Laubichler, "Introductory essay: evolution, comparative biology, and development," in Endothelial Biomedicine, W. C. Aird, Ed., pp. 23-28, Cambridge University Press, Cambridge, UK, 2007.

[25] P. K. Cheruvu, D. Gale, A. M. Dvorak, D. Haig, and W. C. Aird, "Hagfish: a model for early endothelium," in Endothelial Biomedicine, W. C. Aird, Ed., pp. 66-73, Cambridge University Press, Cambridge, UK, 2007.

[26] I. J. McGaw and C. L. Reiber, "Cardiovascular system of the blue crab Callinectes sapidus," Journal of Morphology, vol. 251, no. 1, pp. 1-21, 2002.

[27] I. J. McGaw, "The decapod crustacean circulatory system: a case that is neither open nor closed," Microscopy and Microanalysis, vol. 11, no. 1, pp. 18-36, 2005.

[28] F. R. Schram, Crustacea, Oxford University Press, New York, NY, USA, 1986.

[29] J. L. Wilkens, "The control of cardiac rhythmicity and of blood distribution in crustaceans," Comparative Biochemistry and Physiology Part A, vol. 124, no. 4, pp. 531-538, 1999.

[30] B. R. McMahon and L. E. Burnett, "The crustacean open circulatory system: a re-examination," Physiological Zoology, vol. 63, pp. 35-71, 1990.

[31] C. L. Reiber, B. R. McMahon, and W. W. Burggren, "Cardiovascular functions in two macruran decapod crustaceans (Procambarus clarkii and Homarus americanus) during periods of inactivity, tail flexion and cardiorespiratory pauses," The Journal of Experimental Biology, vol. 200, no. 7, pp. 1103$1113,1997$.

[32] B. R. McMahon, "Control of cardiovascular function and its evolution in Crustacea," The Journal of Experimental Biology, vol. 204, no. 5, pp. 923-932, 2001.

[33] C. L. Reiber, "Hemodynamics of the crayfish Procambarus clarkii," Physiological Zoology, vol. 67, pp. 449-467, 1994.

[34] J. A. Guadagnoli, K. Tobita, and C. L. Reiber, "Assessment of the pressure-volume relationship of the single ventricle of the 
grass shrimp, Palaemonetes pugio," The Journal of Experimental Biology, vol. 210, no. 12, pp. 2192-2198, 2007.

[35] R. E. Shadwick, C. M. Pollock, and S. A. Strickler, "Structure and biomechanical properties of crustacean blood vessels," Physiological Zoology, vol. 63, no. 1, pp. 90-101, 1990.

[36] J. S. Alexandrowicz, "Memoirs: the innervation of the heart of the Crustacea. I. Decapoda," Quarterly Journal of Microscopical Science, vol. 75, no. 298, pp. 181-249, 1932.

[37] T. Kuramoto and A. Ebara, "Neurohormonal modulation of the cardiac outflow through the cardioarterial valve in the lobster," The Journal of Experimental Biology, vol. 111, no. 1, pp. 123-130, 1984.

[38] A. Kihara, K. Kuwasawa, and T. Yazawa, "Neural control of the cardio-arterial valves in an isopod crustacean, Bathynomus doederleini: excitatory and inhibitory junctional potentials," Journal of Comparative Physiology A, vol. 157, no. 4, pp. 529536, 1985.

[39] I. J. McGaw and B. R. McMahon, "Actions of putative cardioinhibitory substances on the in vivo decapod cardiovascular system," Journal of Crustacean Biology, vol. 19, no. 3, pp. 435-449, 1999.

[40] J. L. Wilkens, G. W. Davidson, and M. J. Cavey, "Vascular peripheral resistance and compliance in the lobster Homarus americanus," The Journal of Experimental Biology, vol. 200, no. 3, pp. 477-485, 1997.

[41] J. L. Wilkens and H. H. Taylor, "The control of vascular resistance in the southern rock lobster, Jasus edwardsii (Decapoda: Palinuridae)," Comparative Biochemistry and Physiology Part A, vol. 135, no. 3, pp. 369-376, 2003.

[42] P. G. Bushnell, D. R. Jones, and A. P. Farrell, "The arterial system," in Fish Physiology, W. W. Hoar, D. J. Randall, and A. P. Farrell, Eds., vol. 12a, pp. 89-139, Academic Press, San Diego, Calif, USA, 1992.

[43] W. W. Burggren, "Central cardiovascular function in amphibians: qualitative influences of phylogeny, ontogeny and seasonality," in Mechanisms of Systemic Regulation: Vol. 1. Respiration and Circulation, N. Heisler, Ed., pp. 175-197, Springer, Berlin, Germany, 1995.

[44] D. C. Sandeman, "The vascular circulation in the brain, optic lobes and thoracic ganglia of the crab Carcinus," Proceedings of the Royal Society of London. Series B, vol. 168, no. 10, pp. 82-90, 1967.

[45] J. L. Wilkens, T. Yazawa, and M. J. Cavey, "Evolutionary derivation of the American lobster cardiovascular system: an hypothesis based on morphological and physiological evidence," Invertebrate Biology, vol. 116, no. 1, pp. 30-38, 1997.

[46] R. Pyle and E. Cronin, The General Anatomy of the Blue Crab Callinectes Sapidus Rathbun, State of Maryland Board of Natural Resources Publications no. 87, Chesapeake Biological Laboratory, Solomons Island, Md, USA, 1950.

[47] C. A. Farrelly and P. Greenaway, "The morphology and vasculature of the respiratory organs of terrestrial hermit crabs (Coenobita and Birgus): gills, branchiostegal lungs and abdominal lungs," Arthropod Structure and Development, vol. 34, no. 1, pp. 63-87, 2005.

[48] E. Haeckel, "Über die gewebe des flußkrebses," Archiv für Anatomie, Physiologie und Wissenschaftliche Medizin, vol. 24, pp. 469-568, 1857.

[49] P. T. Johnson, Histology of the Blue Crab. A Model for the Decapoda, Prager Scientific, New York, NY, USA, 1980. 

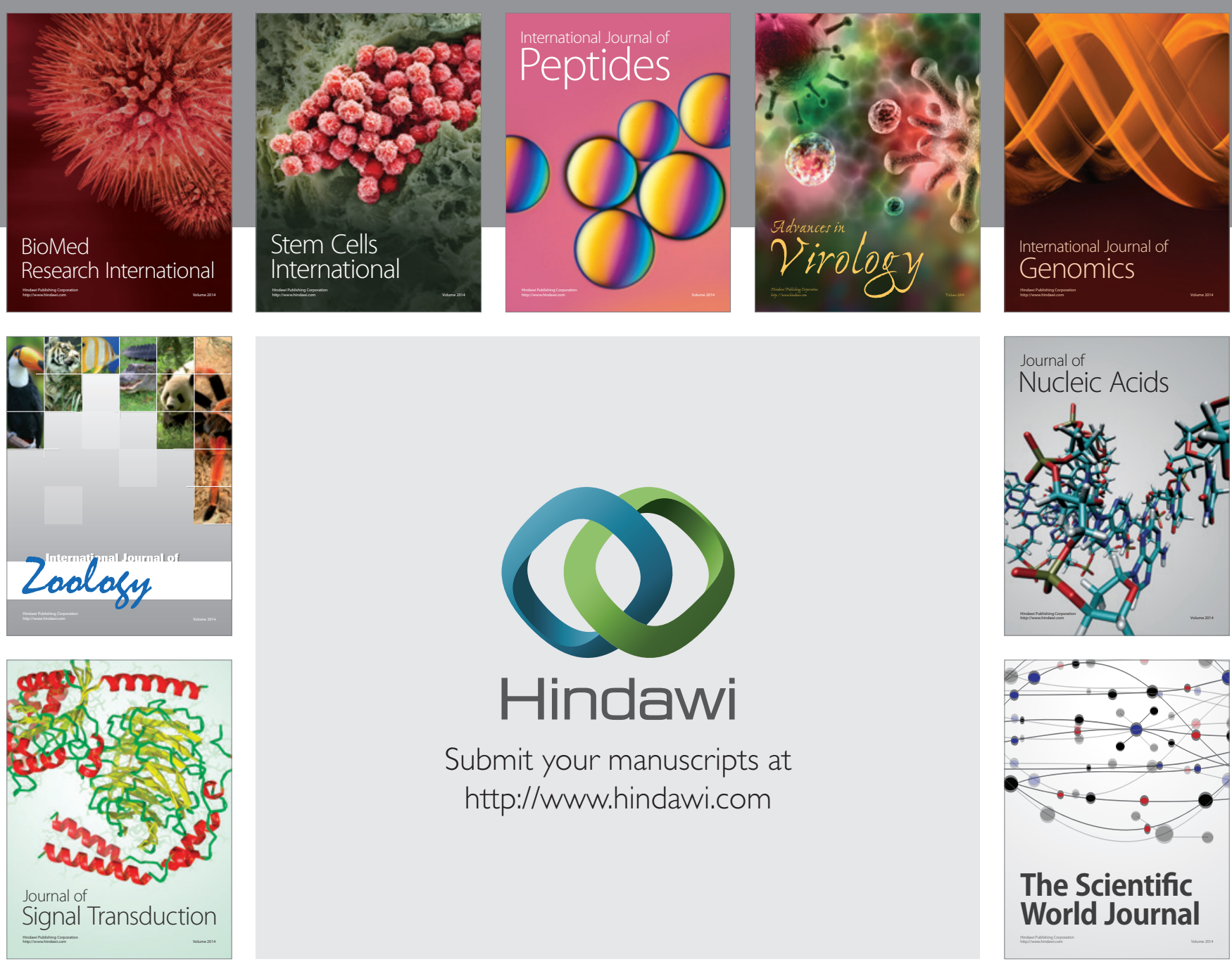

Submit your manuscripts at

http://www.hindawi.com
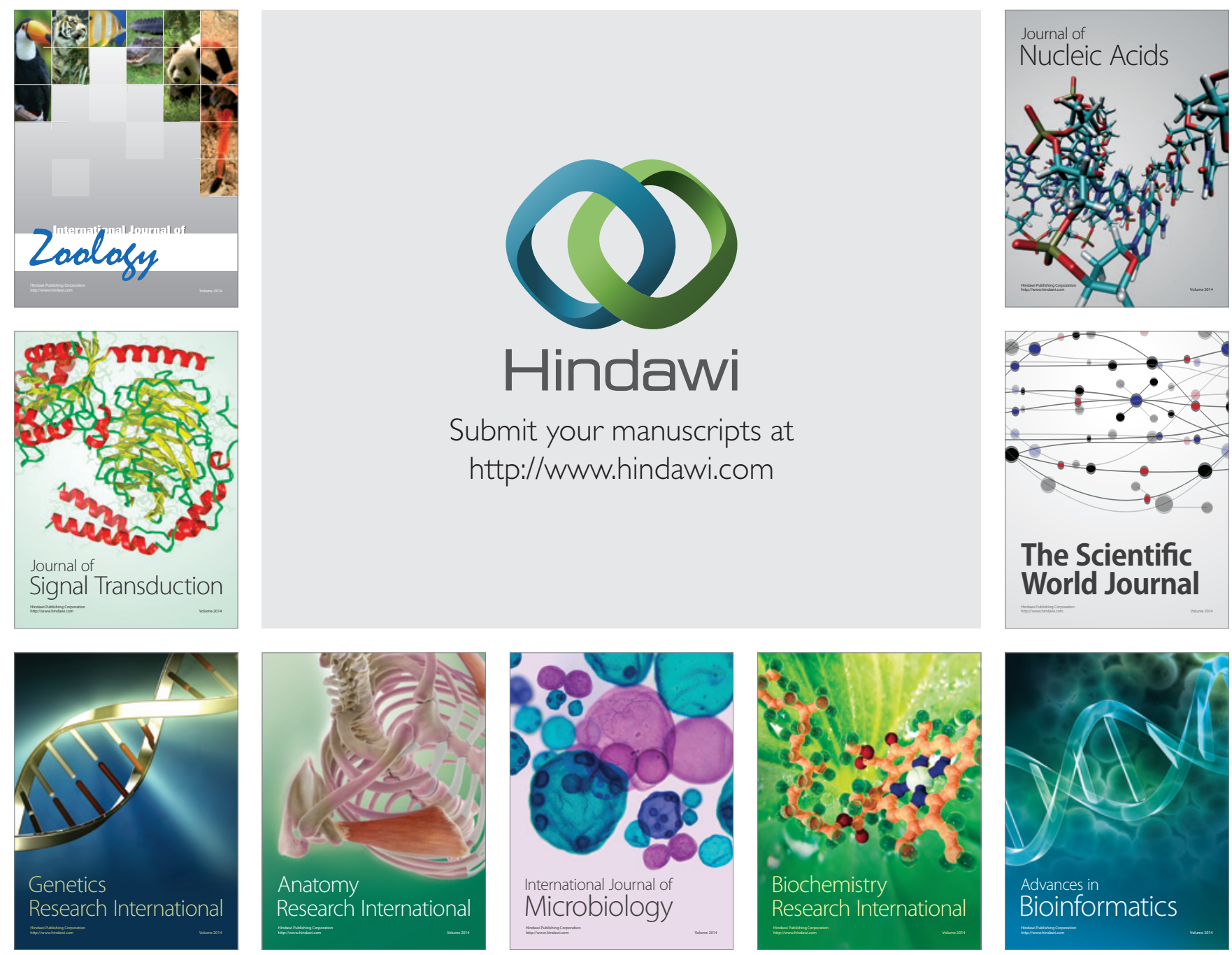

The Scientific World Journal
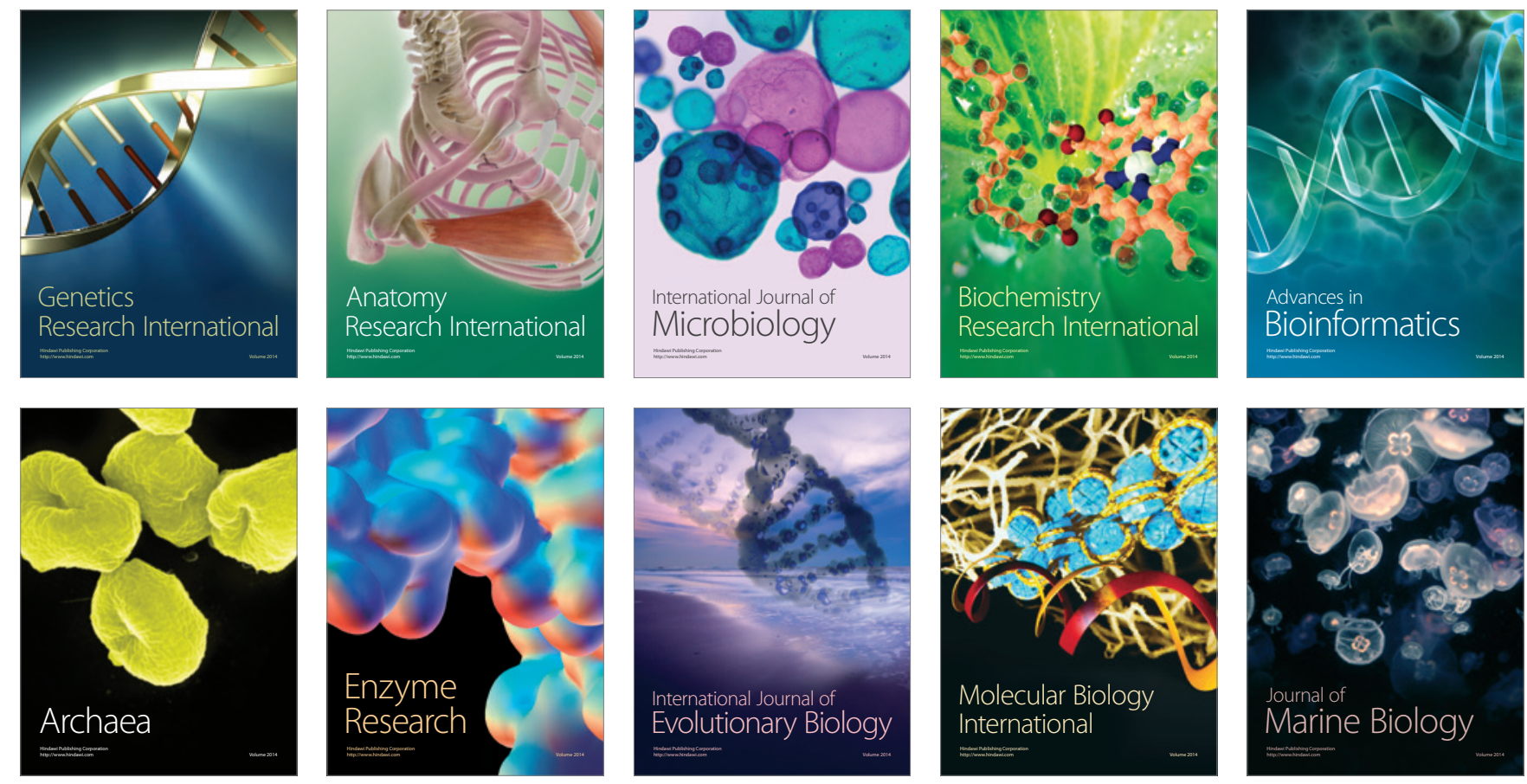